Particle Accelerators, 1990, Vol. 26, pp. 211-216 Reprints available directly from the publisher Photocopying permitted by license only
(C) 1990 Gordon and Breach, Science Publishers, Inc. Printed in the United States of America

\title{
AN 805 MHZ DISK AND WASHER ACCELERATING STRUCTURE WITH COAXIAL COUPLER FOR THE FERMILAB UPGRADE
}

D.E. YOUNG, A. MORETTI, G. LEE, F.E. MILLS, AND P. ZHOU Fermi National Accelerator Laboratory, * Batavia, Illinois D.A. SWENSON, AND P.E. YOUNG

Science Applications International Corporation, Albuquerque, New Mexico

Abstract The Disk and Washer (DAW) structure with four biperiodic tees to support the washers has been studied at Fermilab for the Linac Upgrade by constructing two, $805 \mathrm{MHz}$, one-meter sections joined by a coaxial coupler. The coupler provides an rf drive port, an adjustable tuner, a vacuum port, and an enclosure for the installation of beam focussing and monitoring devices. This configuration is suitable for a high beta proton linear accelerator. The bi-periodic tee supports in the DAW structure suppress the $\mathrm{TM}_{11}$ mode at the operating frequency which has been a problem with previous designs. Frequency-mode spectra have been calculated and measured as well as the quantities $Q, R / Q$, and $2 T^{2}$. The structure has been evacuated and operated at high power levels and high accelerating fields.

\section{INTRODUCTION}

The Disk and Washer (DAW) rf accelerating structure has been studied at Fermilab as a possible structure for extending the energy of the $200-\mathrm{MeV}$ proton linac to $400 \mathrm{MeV} .1$ The DAW structure has the advantage of high efficiency (effective shunt impedance), large coupling between cells, and good vacuum conductance. It suffers from the problem of a multiplicity of other modes in the vicinity of the accelerating mode and the mechanical problem of supporting and cooling the central washer without degrading the rf properties of the structure. The DAW structure has been extensively studied at other laboratories (see reference 2 for citations of early work).

In the Fermilab Linac Upgrade project the beam from the $116 \mathrm{MeV}$ drift-tube linac will be matched into the new rf accelerating structure operating at the higher frequency of $805 \mathrm{MHz}$, and at higher gradient. For the Upgrade the low duty factor simplifies the cooling of the washers in the DAW structure and allows the use of small, biperiodic tee supports. A four-tee configuration will

*Operated by the Universities Research Association under contract with the U.S. Department of Energy. 
introduce a stopband in the TM11-1ike modes which overlap the region of the operating mode frequency. The tees can be designed to present a small perturbation to the accelerating mode by placing and shaping them so as to closely match the null in the accelerating mode field configuration, though they then present a greater perturbation to the structure coupling mode. Thus, it appears that some of the disadvantages of the DAW structure can be overcome in the Fermilab application by developing a design based on washer tee supports following the ideas of Iwashita. 3

\section{DESCRIPTION OF MODELS}

The computer programs SUPERFISH and MAFIA 4 have been used to design a DAW model appropriate for a proton energy of $200 \mathrm{MeV}$. The parameters of this model are shown in Table I. MAFIA was used to calculate the added power losses due to the tees, about $5 \%$. This loss on the tees reduces the shunt impedance (ZTT) by $5 \%$ from the ideal value calculated from SUPERFISH. MAFIA was also used to calculate the full dispersion curve for the structure up to $1.4 \mathrm{GHz}$, Fig. 1. The biperiodic tee supports produce a stopband in the TM11 mode centered near the operating frequency of the accelerating $\mathrm{TM}_{02} \pi$ mode. The biperiodic tees have produced stopbands in most of the modes with the one exception being the $\mathrm{TM}_{02}$ mode because of the placement of the tee supports in the null of this mode.

\begin{tabular}{cc} 
TABLE I Nominal DAW cavity & parameters \\
\hline Frequency $(\mathrm{MHz})$ & 805 \\
Cell length $(\beta=0.5662) \mathrm{cm}$ & 10.544 \\
Cavity radius $(\mathrm{cm})$ & 27.220 \\
Disk radius (cm) & 20.119 \\
Half disk thickness (cm) & 2.672 \\
Tee-support tubing $0 . \mathrm{D} .(\mathrm{cm})$ & 0.5 \\
Washer radius $(\mathrm{cm})$ & 17.604 \\
Half washer thickness (cm) & 0.63 \\
Nose angle & $30^{\circ}$ \\
Nose radius (cm) & 0.5 \\
Bore radius $(\mathrm{cm})$ & 1.5 \\
Gap length $(\mathrm{cm})$ & 4.23 \\
ZTT (single cel1) $\mathrm{MD} / \mathrm{m}$ & 45.9 \\
\hline
\end{tabular}

To study the DAW structure we have built a number of assemblies which can be configured in different ways, as shown in Fig. 2. The parts consist of two one-meter, ten-cell cavities, 
one with power capability and one demountable, a coaxial bridge coupler with a tuner and wave guide rf port, 5 a set of end plates for terminating either the coupling or the accelerating modes, and demountable disks for extra tuning capability. For example, the use of a pair of removable disks and a pair of coupling mode terminators on the one meter DAW sections allows an investigation of the coupling mode. The use of the pair of accelerating mode terminations allows study of the accelerating mode and all other modes which may be present. In a similar manner the bridge coupler can be studied by itself or configured with the other structures.

The one-meter section and coaxial bridge coupler were constructed so that power tests, under vacuum, could be performed with water cooling adequate for a $0.2 \%$ duty factor. The washers were machined from OFE class II copper plate. A cooling channel was machined in the plate and covered with a copper ring with flared stainless-steel adapters for connecting the tee supports. The disks were made of aluminum and plated with $0.005 \mathrm{~cm}$ of copper. The tees are copper plated stainless steel tubing. Bimetallic aluminum stainless steel struts are press-fitted into the disks and used to connect the tee supports for the washers. The disk-and-washer assembly is shrunk fit into the copper-plated aluminum tanks. An aluminum weld from the tank outside makes a water tight, vacuum tight joint between the outside cylinder and the struts. Water cooling is provided through channels on the outer tank wall to each washer with two tees carrying the water in and two tees carrying the water out. Vacuum/rf flanges are welded on the ends of the tanks to accept the end plate terminators or the bridge coupler for rf power testing. The bridge coupler contains the rf input wave-guide port, rf tuner and the vacuum pumpout port. A photograph of this power model is shown in Fig. 3 .

\section{RESULTS}

The structure must be tuned so that the accelerating mode and the coupling mode occur at the same frequency at the confluence of the $\mathrm{TM}_{01}$ and $\mathrm{TM}_{02}$ passbands. This is done by determining dimensions from 
one, two, or three cell configurations where either the accelerating or coupling modes could be measured independently. This procedure allowed the ten-cell cavity to be assembled so that its frequency was about $100 \mathrm{kHz}$ below the desired value and well within the tuning range of the end-cell tuners. The field pattern was measured by pulling a metallic bead at a fixed frequency and measuring the phase change of the cavity field with a network analyzer. Fig. 4 shows the measured fields on the axis of the ten-cell with the coaxial coupler attached and tuned. In this figure the ordinate is proportional to the square root of the electric field in the accelerating gap. The structure was found to be extremely stable with a coupling constant of about $30 \%$ with very little tilt. The cell-to-cell field variations are less than $1.5 \%$ and due mainly to cell-to-cell geometry changes of \pm 0.050 cms .

The dispersion curve, Fig. 1, was calculated using the MAFIA program. There was good agreement between the calculated dispersion curve and the measurements in the ten-cell cavity over the passband of the TM01 mode. A preliminary check of other modes also showed good agreement. However, two satellite resonances were found near the $\pi$ accelerating mode frequency which had the symmetry of the TE31 mode; one with a field null on the vertical tees, the other on the horizontal tees. Using the tuner provided in the coaxial coupler, the $\pi$-mode resonance could be moved away from the $\mathrm{TE}_{31}$ resonances, thus allowing the $Q$ of the structure to be measured without interference effects. $R / Q$ (shunt impedance/quality factor) was measured using a bead-pull on the beam axis which agreed to within $1 \%$ of the value obtained from SUPERFISH. The results of the $R / Q$ and $Q$ measurements for the ten-cell and ten-cell with coaxial bridge coupler are given in Table II.

\begin{tabular}{lcc} 
TABLE II Measured properties of the structure & of \\
& $\begin{array}{c}\text { Ten-cell } \\
\text { mode1 }\end{array}$ & $\begin{array}{c}\text { Ten -cell model } \\
\text { w/coax coupler }\end{array}$ \\
\hline R/Q & 180.72 & 176.63 \\
Q (from SUPERFISH) & 37,260 & 34,213 \\
Q (measured) & 28,600 & 26,400 \\
ZTT (measured) M/m & 41.7 & 37.0 \\
\hline
\end{tabular}

The ten-cell cavity with coaxial bridge coupler was powered with a $1.25 \mathrm{MW}, 805 \mathrm{MHz}$, klystron-based power supply at a $0.1 \%$ 
duty factor, i.e. $7.5 \mathrm{~Hz}$ repetition rate and $120 \mu \mathrm{sec}$ pulse. This power level resulted in a peak axial field of $7.2 \mathrm{MV} / \mathrm{m}$ and a maximum surface field of $32.0 \mathrm{MV} / \mathrm{m}$. The structure operated reliably at this power level after a short period of voltage conditioning.

\section{CONCLUSION}

These model experiments have shown that the DAW structure with biperiodic tee supports for the washers is a viable candidate for ion acceleration following the drift-tube structure in a linac, especially for low duty factor applications. In this case the small bi-periodic supports suppress the troublesome $\mathrm{TM}_{11}$ modes and allow adequate cooling of the washers without degrading the high efficiency of the structure. The high coupling constant of the structure allows a greater tolerance for errors as well as greater stability and power flow. In the particular model studied, difficulties were experienced due to the structural integrity and lack of rigidity of the supports. Further development is required to insure against failure of the numerous water-to-vacuum joints which would be difficult to repair. The coaxial bridge coupler design used in this model has the advantage of breaking up the structure and inhibiting the propagation of undesireable modes. In a linac with many sections, it is important to measure the modes in each section separately and to tune away any modes found to interfere with the accelerating mode as was necessary in this experiment.

\section{REFERENCES}

1. D.E. Young $400 \mathrm{MeV}$ Upgrade for the Fermilab Linac, Particle Accelerators, (Gordon and Breach), these proceedings.

2. A. Moretti, et a1, An $805 \mathrm{MHz}$ Disk and Washer Structure for the Fermilab Upgrade, Proc. of the 1988 Linear Accelerator Conference, CEBAF (to be published).

3. Y. Iwashita, et a1, $Q$ Value of DAW Linac with Biperiodic $T$ Stem, Proc. of the 1986 Linear Accelerator Conference, SLAC Report 303, pgs. 151-153 (1986).

4. T. Weiland, et al, MAFIA - A Three Dimensional Electromagnetic CAD system for Magnets, RF Structures, and Transient Wake-field Calculations, 1986 Linear Accelerator Conference, SLAC Report SLAC-303, 276-278 (1986).

5. A.G. Daikovsky, et al, "Coaxial Couplers in Disk-and-Washer Structures", Particle Accelerators, 15, pgs. 209-219, (1984) 

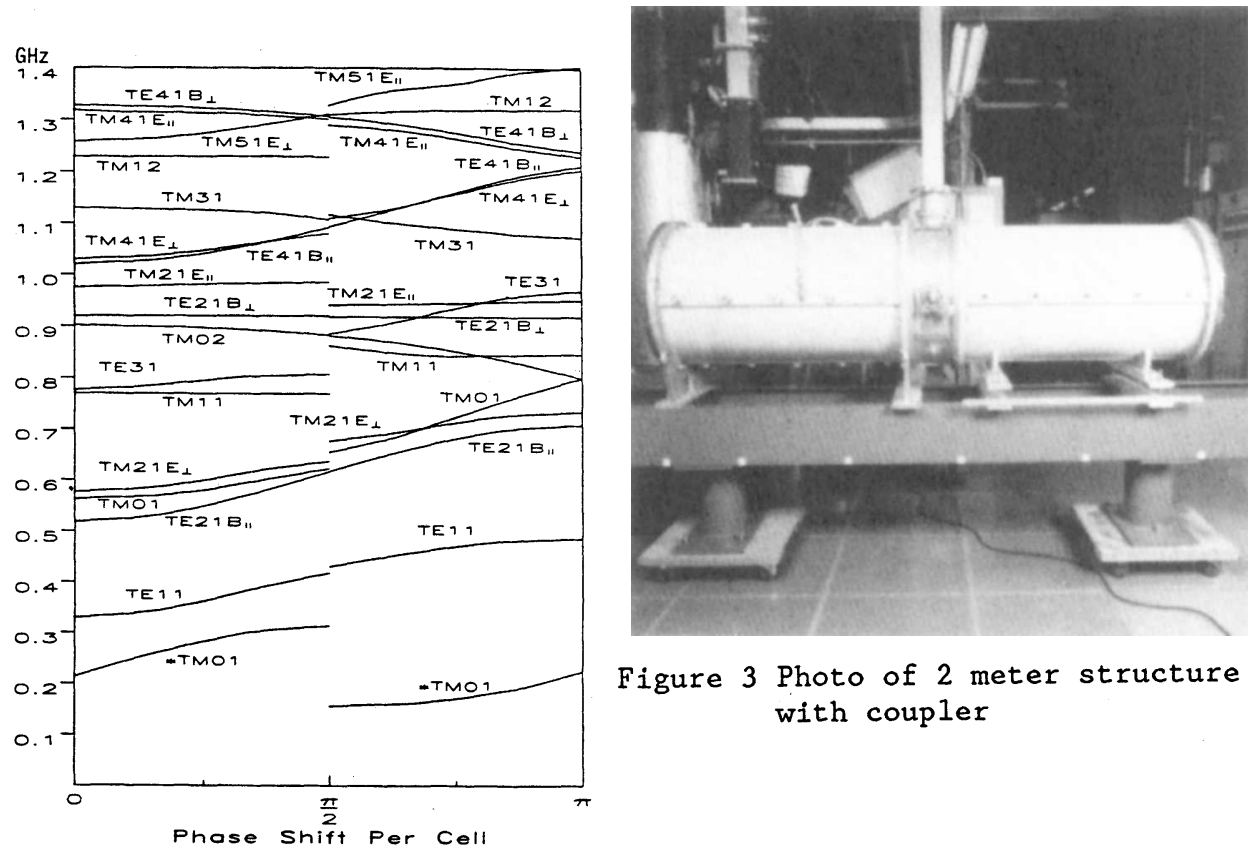

Figure 3 Photo of 2 meter structure with coupler

Figure 1 DAW dispersion curve
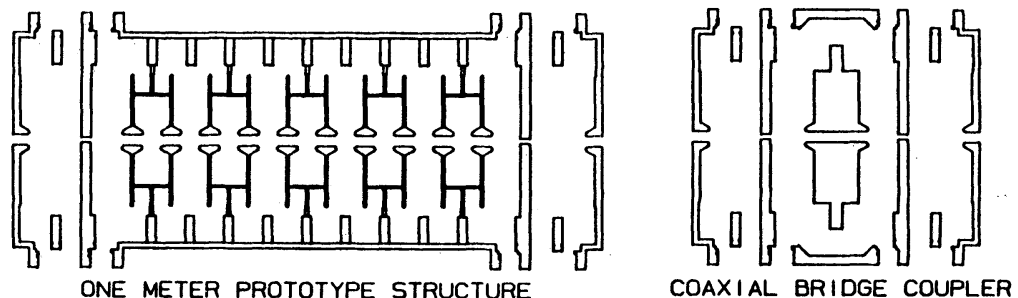

Figure 2 DAW configurations

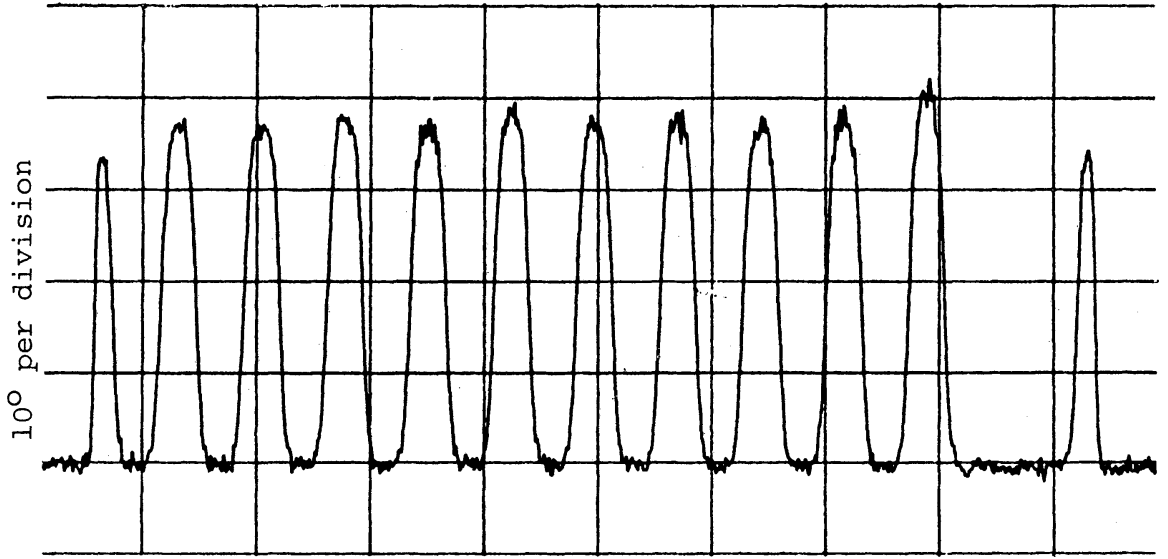

Relative Axial Position

Figure 4 Measured axial fields of model (with coupler) 